\title{
Individual differences and memory for faces, pictures, and words
}

\author{
M. M. WOODHEAD and A. D. BADDELEY \\ M.R.C. Applied Psychology Unit, Cambridge CB2 2EF, England
}

\begin{abstract}
People who had performed exceptionally well or badly on facial recognition tests between 1 and 4 years previously were given three recognition tasks, one involving faces, one involving paintings, and a third using visually presented words. Those selected as good recognizers were better on the recognition of faces and paintings, but they did not differ in verbal memory. This result suggests that people who are particularly good at facial recognition have a generally good visual memory that is separate from verbal memory.
\end{abstract}

There has in recent years been considerable discussion on the question of whether visual and verbal long-term memory rely on different systems, as the dual coding hypothesis of Paivio (1971) would suggest, or whether it is more economical to explain both types of memory in terms of a single abstract propositional system (Anderson \& Paulson, 1978). Within visual memory, a similar controversy has concerned the question of whether memory for faces involves a system separate from other aspects of visual memory (Ellis, 1975). While it is doubtful whether any single experiment or, indeed, sequence of experiments can decide between these various viewpoints, one can usefully explore the question of the extent to which these three types of memory appear to behave in similar ways. One approach involves carrying out parallel experiments and investigating whether the same kind of variable influences verbal, pictorial, and face memory. A second approach is to study the breakdown of such memory systems in brain-damaged patients, asking whether a subject whose performance on one type of material is disrupted will necessarily perform badly on the other two. The approach adopted in this study is a third one, that takes advantage of individual differences in performance.

Subjects are selected on the basis of being very good or very bad at face recognition, and their performance on a task involving memory for paintings and memory for words is then studied. If all three types of memory rely on a homogeneous abstract memory system, then one might expect those people who are good at remembering faces also to be good at remembering paintings and words. On the other hand, if visual and verbal memory in some sense utilize different systems, one might expect people who are good at remembering faces also to be good at remembering paintings, but to have no advantage in remembering words. However, if people selected as good at remembering faces are not especially good at remembering either paintings or words, this

We wish to express our thanks to Ian Nimmo-Smith for his advice on data analysis. would lend support to the view that there is a specific capacity for facial memory that is separate from other aspects of human memory. In the present study, therefore, we took advantage of having previously tested several hundred members of our subject panel on facial recognition. We selected subgroups of very good face recognizers and very poor face recognizers and retested them, studying their performance on faces, paintings, and words.

\section{METHOD}

\section{Subjects}

The critical element in selecting hypothetically good and bad recognizers was based on the experimental records of a voluntary subject panel of local residents. Approximately 400 members of this panel had participated one or more times between 1975 and 1978 in six investigations of facial recognition, and their test data were on file. Some 260 of these members were still serving on the panel. Previous recognition performances had been scored for discriminability by $\mathrm{d}^{\prime}$ measures, which took account of the number of false detections relative to the number of correct recognitions. It was the extreme scorers on the d' measure, irrespective of which of the recognition tests they had originally performed, whom it was now proposed to label good or bad recognizers and investigate further. The 40 highest and 40 lowest scorers were contacted in 1979 by the routine system, with no mention of the underlying reason. Approximately half accepted, from which it was possible to form an experimental group of 19 high and 19 low scorers. The previous records of $\mathrm{d}^{\prime}$ recognition scores for these subjects ranged from 2.93 to 6.8 for good recognizers and from -.5 to .92 for bad recognizers. (The nonextreme subjects who were not contacted had all scored in the range of 1.0 to 2.89.)

The previously good recognizers became Group G, the previously bad recognizers became Group B, and all remained unaware of any distinction. In Group $G$ there were 6 men and 13 women (age range $20-60$ years, mean age 40 years, standard deviation 10.1). Group B consisted of 5 men and 14 women (age range 32-58 years, mean 49 years, standard deviation 8.5 ). The difference in ranges was mainly due to one 20-year-old male in Group G; otherwise, the range for that group was $30-60$ years. The varying backgrounds and education of the subjects were representative of the local population.

\section{Materials and Procedure}

Three tests, faces, paintings, and words, were adapted from the tests in an experiment that Warrington (1974) conducted with neurological patients, who were individually tested and 
allowed to respond in their own time. In the present study, the subjects were tested in groups of three to six in time-controlled conditions. The test sessions for $G$ and $B$ subjects were conducted separately to allow planned posttest discussions with the experimenter. The possibility of experimenter bias due to separating the $G$ and $B$ sessions was preferred to the problem of unknown bias if hypothetically very good and very bad subjects participated together in the posttest discussions.

Each of the three tests comprised slides of 100 items, of which 50 were targets and 50 were distractors. The stimuli in the faces test consisted of black-and-white photographs of the faces, including neck area, of unfamiliar actors whose names were unlikely to be known to the public. The paintings test consisted of representational 19 th and 20 th century paintings, mainly scenes and objects; a few contained human figures, but these were not conventional portraits. The words test was composed of commonly used three-, four-, and five-letter nouns, verbs, and adjectives. The order of test presentation to groups of subjects was controlled by a Latin square design.

The test procedure began with the presentation of 50 consecutive target items at a 5 -sec rate. The subjects were instructed to look at each item and decide individually whether it was pleasant or unpleasant, marking $\mathrm{P}$ or $\mathrm{U}$ against the appropriate item number on a response form. After collection of the forms, the test continued with another 50 slides at a 5 -sec rate, each displaying one target and one distractor, side by side. Targets were distributed equally of ten on the left and right sides of distractors. They were presented in a random order that differed from the present presentation. In the paintings test, a target was always paired with a distractor by the same artist and had similar form and/or color. In the case of the faces test, there was some degree of homogeneity within each pair. In the words test, the words were paired at random. Next to each item number on the response form was a double box, and subjects were instructed to check the left or right box according to their decision that the target was on the left or right of the display, guessing if unsure.

The completed test was followed by an informal discussion on reactions to the three tasks. The experimenter used a protocol of questions as a guide, including the relative difficulties of the tests and the strategies used to memorize the targets; she openly took written notes of the views expressed by each subject.

\section{RESULTS}

A comparison of the two groups on the orienting task of judging the pleasantness of the test items showed no difference between the groups, and this variable was not considered further.

The mean error scores on the recognition tests appear in Table 1. The standard of performance of Group G was very high, and it seemed appropriate to apply a

Table 1

Mean Error Scores on Three Recognition Tests for Subjects Classified as Good or Bad Recognizers on the Basis of Previous Tests

\begin{tabular}{lccccc}
\hline & \multicolumn{2}{c}{$\begin{array}{c}\text { Group G } \\
\text { Good Subjects }\end{array}$} & & \multicolumn{2}{c}{$\begin{array}{c}\text { Group B } \\
\text { Bad Subjects }\end{array}$} \\
\cline { 2 - 6 } \multicolumn{1}{c}{ Test } & Mean & SD & & Mean & SD \\
\hline Faces & 1.9 & 2.6 & 7.3 & 6.0 \\
Paintings & 0.6 & 0.8 & & 2.4 & 2.6 \\
Words & 2.0 & 1.8 & & 2.1 & 2.2 \\
\hline
\end{tabular}

Note $-N=19$ for both conditions. transformation of the data (of both groups) in order to achieve a greater homogeneity of variance. An arcsin square root transformation was therefore applied prior to performing analysis of variance. A significant difference was found between good and bad recognizers $[F(1,36)=16.31, p<.001]$, an overall difference between the three tests $[F(2,72)=13.1, p<.001]$, and an interaction between the groups and the tests $[F(2,72)=9.07, \quad p<.001]$. Multiple comparisons (Newman-Keuls test) showed that for Group G there were no differences between the three tests. For Group B, however, although the paintings and words tests did not differ, both were performed significantly better than was the faces test $(\mathrm{p}<.001)$. The comparisons between the groups revealed that Group $G$ performed better than Group B on faces $(p<.001)$ and on paintings $(p<.05)$ but did not differ on the words test $(p=.92$, two-tailed). This lack of difference for words suggests that the verbal encoding ability of the subjects was independent of their ability to recognize pictorial material. A similar effect was noted by Goldstein and Chance (1970). Their subjects were asked to give verbal associates to pictures of inkblots, snow crystals, and faces, some of the stimuli in each of these classes having emerged as highly recognizable in another experiment with different subjects. No positive relationships were found between that recognizability and the new verbal associations. It appears that the analysis of Goldstein and Chance, which takes advantage of differences between items, reaches the same conclusion as our own study, which takes into account the difference between subjects. Both results imply separation between visual and verbal memory.

In the discussion immediately following the completion of the tests, Group $G$ reported a total of 83 ways of trying to memorize the test items, and Group B reported 77 . The methods were categorized under 11 strategies, shown in Table 2 . The only statistically reliable differences in strategies between the groups was the selection of features as memory cues. This strategy produced an interesting anomaly. In the faces test, the good recognizers tended to make more use of feature selection than the bad recognizers did $\left(\chi^{2}=4.21\right.$, $\mathrm{p}<.05$ ), but the effect was reversed in the paintings test, for which more feature selection was reported by bad recognizers than by good recognizers $\left(\chi^{2}=4.61\right.$, $\mathrm{p}<.05)$. It appears that when Group B used feature selection as a recognition strategy, it was only minimally for faces.

This pattern of results might suggest that instructions to select features would enhance memory for faces. However, as Patterson and Baddeley (1977) have shown, a feature strategy appears to lead to performance poorer than that resulting from a strategy based on such general judgments as intelligence or friendliness. Bearing this in mind, it would seem unwise to draw firm conclusions from the subjective report data. 
Table 2

Recognition Strategies Reported by Subjects

\begin{tabular}{lrrrrrrr}
\hline & \multicolumn{3}{c}{ Subjects in Group G } & \multicolumn{3}{c}{ Subjects in Group B } \\
\cline { 2 - 4 } \cline { 6 - 8 } \multicolumn{1}{c}{ Strategy } & F & \multicolumn{1}{c}{ P } & W & F & P & W \\
\hline General Impression & 10 & 7 & $*$ & 10 & 4 & $*$ \\
Feature Selection & 10 & 2 & $*$ & 3 & 9 & $*$ \\
Character Inference & 8 & 1 & $*$ & 3 & 0 & $*$ \\
Labels and Names & 6 & 1 & $*$ & 5 & 3 & $*$ \\
Facial Expression & 5 & 0 & $*$ & 6 & 0 & $*$ \\
Clothing & 3 & 0 & $*$ & 1 & 0 & $*$ \\
Associations & 0 & 0 & 14 & 4 & 0 & 10 \\
Color Cues & $*$ & 11 & $*$ & $*$ & 14 & $*$ \\
Style & $*$ & 2 & $*$ & $*$ & 2 & $*$ \\
Meaningfulness & $*$ & 0 & 3 & $*$ & 0 & 2 \\
Sound of Words & $*$ & $*$ & 0 & $*$ & $*$ & 1 \\
Totals & 42 & 24 & 17 & 32 & 32 & 13 \\
\hline
\end{tabular}

Note $-F=$ faces, $P=$ paintings, and $W=$ words.

* Not applicable.

\section{DISCUSSION}

Our results show, first of all, that it is possible to isolate groups of people who are consistently good or consistently bad at recognizing photographs of faces. This suggests, in short, that our test of face memory is a reasonably reliable one. Subjects who are good at recognizing faces also appear to be good at recognizing paintings of objects and scenes, being consistently superior on this task to subjects who are poor at recognizing faces. Such a result implies that memory for faces and memory for pictures may well involve broadly similar memory capacities, and, as such, it supports the contention of Ellis (1975) that facial memory has not been convincingly shown to depend on a specific and separate memory system.

The fact that people who are good at remembering faces are also good at remembering paintings might simply imply an overall memory ability, perhaps based on general intelligence. Results of the verbal recognition task, however, allow us to reject this interpretation, since the two groups show absolutely no difference in memory for words. Our results, therefore, are clearly consistent with the view that visual and verbal memory involve separate systems. It is tempting to conclude that the two systems use separate data bases. While this is certainly a strong possibility, our results do not allow us to locate the difference within the memory system with any great confidence. The absence of any clear difference in reported strategy does, however, argue against an interpretation based on initial processing differences. The use of a two-alternative forced-choice recognition procedure for testing in all three cases makes it difficult to think of a convincing interpretation of the visual/ verbal difference in terms of retrieval strategy, leaving a storage interpretation the most obvious account of the difference between visual and verbal recognition.

Suppose we do assume that storage differences occur, do they imply separate systems? While they suggest a distinction between visual and verbal memory, our results could be interpreted either in terms of quite separate storage systems or in terms of two different types of association within a single system. How could one decide between these two? One source of evidence might come from memory disruption in brain damage. The evidence here is equivocal; globally amnesic patients seem to show disruption of both verbal and nonverbal memory (e.g., Warrington, 1974). On the other hand, there is no doubt that short-term visual and short-term verbal memory may be separately disrupted by brain damage (Shallice, 1979; Warrington, 1971), and there is evidence to suggest that damage to the left hemisphere will impair verbal learning, whereas right-hemisphere damage disrupts performance on more visual tasks (Milner, 1971). However, the verbal/visual differences appear to be much less clear-cut in long-term learning than they are in performance on immediate memory tasks, and it is possible that those effects that do occur result from defective input processing rather than from disruption of a specific visual or verbal long-term store. In conclusion, then, it appears that although our data strongly support the view that visual and verbal memory are separable, it is still unclear whether this implies two storage systems or merely two types of association within a common system.

\section{REFERENCES}

Anderson, J. R., \& Paulson, R. Interference in memory for pictorial information. Cognitive Psychology, 1978, 10, 178-202.

Ellis, H. D. Recognizing faces. British Journal of Psychology, $1975,66,409-426$.

Goldstein, A. G., \& Chance, J. E. Visual recognition memory for complex configurations. Perception \& Psychophysics, 1970, 9, 237-241.

Milner, B. Interhemispheric differences and psychological processes. British Medical Bulletin, 1971, 27, 272-277.

PAIvio, A. Imagery and verbal processes. New York: Holt, Rinehart, \& Winston, 1971.

Patterson, K. E., \& Baddeley, A. D. When face recognition fails. Journal of Experimental Psychology: Human Learning and Memory, 1977, 3, 406-417.

Shallice, T. Neuropsychological research and the fractionation of memory systems. In L. G. Nilsson (Ed.), Perspectives on memory research. Hillsdale, N.J: Erlbaum, 1979.

Warrington, E. K. Neurological disorders of memory. British Medical Bulletin, 1971, 27, 243-247.

Warrington, E. K. Deficient recognition memory in organic amnesia. Cortex, 1974, 10, 289-291.

(Received for publication November 7, 1980; revision accepted February 4,1981 .) 